\title{
IMPACT OF FECAL CALPROTECTIN MEASUREMENT FOR THE DIAGNOSIS OF INFLAMMATORY BOWEL DISEASE IN CHILDREN WITH ALARM SYMPTOMS
}

\author{
BURCU GÜVEN ${ }^{1}$, fatma İssi ${ }^{1}$, Elif Sağ ${ }^{1}$, and Kurtuluş Buruk ${ }^{1}$ \\ ${ }^{1}$ Affiliation not available
}

September 17, 2020

\begin{abstract}
Background: Discrimination of inflammatory bowel diseases (IBD) and other gastrointestinal diseases in pediatric patients is highly important and the definitive diagnosis of IBD is established by endoscopic examination. the use of noninvasive methods (clinical symptoms and laboratory tests) allows an early and accurate referral of patients from first step health centers to advanced health centers. In the present study, we aimed to investigate the effectiveness of fecal calprotectin (FC) in the discrimination of IBD and other gastrointestinal diseases in children. Methods: The retrospective study included the patients that underwent FC testing and colonoscopy. Demographic characteristics, Alarm symptoms (AS), and abnormal laboratory findings (ALF) were recorded for each patient. A negative calprotectin result was considered to be less than $50 \mu \mathrm{g} / \mathrm{g}$, and a second cutoff value for FC was accepted as $150 \mu \mathrm{g} / \mathrm{g}$. Definitive diagnosis was established by colonoscopy in each patient. Results: The study included 88 consecutive patients (mean age, $10.2 \pm 6.1$ years; $51.1 \%$ female). Of these, 20 (22.7\%) patients were diagnosed with IBD. No significant difference was found between IBD and non-IBD patients with regard to AS except for involuntary weight loss $(\mathrm{p}=<0.001)$. The incidence of increased CRP and hypoalbuminemia was significantly higher in IBD patients $(\mathrm{p}=0.002$ and $\mathrm{p}=0.026$, respectively). AS combined with ALF and FC $>150 \mu \mathrm{g} / \mathrm{g}$ had the highest specificity $(95.12 \%)$. Conclusions: Although primary care clinicians often use AS and laboratory parameters in the discrimination of IBD and non-IBD diseases, FC was found to have a relatively higher diagnostic value.
\end{abstract}

\section{Introduction}

Discrimination of inflammatory bowel diseases (IBD) and other gastrointestinal diseases in pediatric patients is highly important and the definitive diagnosis of IBD is made by endoscopic and histopathological examinations. Although the definite diagnosis of IBD is established by colonoscopy and histopathologic examinations, the use of noninvasive methods (clinical symptoms and laboratory tests) allows an early and accurate referral of patients from family physicians or general pediatricians to pediatric gastroenterology centers. Additionally, the use of these methods will also be helpful in the differentiation of IBD from other organic diseases such as polyps, solitary rectal ulcer, and allergic colitis (1).

Fecal calprotectin (FC), which is a neutrophil-derived protein released in stool in response to mucosal inflammation, has recently emerged as a practical, simple, and noninvasive test in the diagnosis of IBD. FC plays an immunoregulatory role in interaction with the zinc-dependent metalloproteinases responsible for the activation of proinflammatory cytokines. Moreover, its fecal excretion is highly correlated with the severity of intestinal inflammation (2). FC is resistant to colonic bacterial degradation and can be stored at $-20{ }^{\circ} \mathrm{C}$ without decomposition and is stable for up to seven days at room temperature, which increase its use in clinical practice (3). Nevertheless, its sensitivity is remarkably high (usually $100 \%$ at $<50 \mu \mathrm{g} / \mathrm{g}$, ranging from $83 \%$ to $100 \%$ at $50 \mu \mathrm{g} / \mathrm{g})$ since it has a broad range $(0-3000 \mu \mathrm{g} / \mathrm{g})$ and increases as a result of numerous factors including celiac disease, infectious gastroenteritis, and the use of proton pump inhibitors 
and nonsteroidal anti-inflammatory drugs (4-6). Moreover, its specificity ranges between $51 \%$ and $100 \%$ (6), which is relatively lower in children (7) and thus additional findings are needed to enhance the specificity in children.

In the present study, we aimed to investigate the effectiveness of FC measurement in the discrimination of IBD and other colonic diseases in children.

\section{Materials and Methods}

The retrospective study included 88 patients that presented to Karadeniz Technical University Medical School Pediatric Gastroenterology outpatient clinic with gastrointestinal symptoms and underwent colonoscopy with a pre-diagnosis or exclusion of organic gastrointestinal disease and had FC measurement. Demographic characteristics, AS, and ALF were recorded for each patient. AS included rectal bleeding, involuntary weight loss, chronic diarrhea, perianal lesions, extraintestinal findings, and family history of IBD $(8,9)$. ALF included anemia, hypoalbuminemia $(<3.5 \mathrm{~g} / \mathrm{dL})$, increased erythrocyte sedimentation rate (ESR) $(>20$ $\mathrm{mm} / \mathrm{sec})$, and increased C-reactive protein $(\mathrm{CRP})$ level $(>1 \mathrm{mg} / \mathrm{dL})$, thrombocytosis $(>450,000 / \mu \mathrm{L})(10)$. Anemia was defined as a hemoglobin $(\mathrm{Hb})$ level of $>2$ standard deviations $(\mathrm{SD})$ from the mean for age and gender for the entire population (8).

Concentration of FC in stool samples was measured semi-quantitatively by using a CalFast XT immunochromatographic assay with a mixture of anticalprotectin monoclonal and polyclonal antibodies (Eurospital, Trieste, Italy). A negative calprotectin result was considered to be less than $50 \mu \mathrm{g} / \mathrm{g}(4)$, and a second cutoff value for $\mathrm{FC}$ was accepted as $150 \mu \mathrm{g} / \mathrm{g}$ (6). Its range changed 0 to $300 \mu \mathrm{g} / \mathrm{g}$. Definitive diagnosis of colonic disease was established by colonoscopy and histpathological examination in each patient.

Statistical analysis

Data were analyzed using SPSS version 21.0 (IBM Corp. Released 2012. IBM SPSS Statistics for Windows, Version 21.0. Armonk, NY: IBM Corp.). Quantitative variables were expressed as mean, standard deviation (SD), and minimum-maximum values. Categorical variables were expressed as frequencies (n) and percentages (\%). The sensitivity, specificity, positive predictive value (PPV), negative predictive value (NPV) and accuracy were calculated for the diagnosis of IBD in patients with AS and FC using binary logistic regression. The Area under the Receiver Operating Characteristic curve (AUC) was used for assessing the value of AS and FC in the prediction of IBD in children with gastrointestinal symptoms.

The study was approved by Karadeniz Technical University Medical School Ethical Committee (Approval No: 2020-127).

\section{Results}

The study included 88 consecutive patients (mean age, $10.2 \pm 6.1$ years; range, 2 months - 18 years; $51.1 \%$ female). Of these, $20(22.7 \%)$ patients were diagnosed with IBD, including $12(13.6 \%)$ patients with ulcerative colitis (UC) and 8 (9.1\%) patients with Crohn's disease (CD). The remaining 68 (77.3\%) patients had no IBD, including $16(18.2 \%)$ patients with lymphonodular hyperplasia, 11 (12.5\%) patients with non-specific colitis and $10(11.4 \%)$ patients with allergic colitis. Colonoscopy was normal in 31 (35.2\%) patients (Figure 1).

Alarm symptoms (AS) were present in $55(62.5 \%)$ patients and the most common alarm symptom was rectal bleeding ( $\mathrm{n}=37 ; 42.0 \%)$, followed by involuntary weight loss $(\mathrm{n}=31 ; 35.2 \%)$, family history of IBD $(\mathrm{n}=7 ; 7.79 \%)$ and perianal lesions $(\mathrm{n}=3 ; 3.4 \%)$ (Table I). No extraintestinal symptoms were detected in any patient. No significant difference was found between IBD and non-IBD patients with regard to AS except for involuntary weight loss, which was significantly greater in IBD patients compared to non-IBD patients (75.0 vs $23.5 \%, p=<0.001$, OR: $9.75,95 \%$, CI 3.0 to 31.00 ) (Table I).

Abnormal laboratory findings (ALF) were present in 38 (43.2\%) patients and the most common abnormal laboratory finding was increased CRP $(\mathrm{n}=15 ; 17.0 \%)$, followed by increased ESR $(\mathrm{n}=12 ; 13.6 \%)$, hypoalbuminemia $(\mathrm{n}=9 ; 10.2 \%)$, and anemia $(\mathrm{n}=8 ; 9.1 \%)$ (Table I). The incidence of increased CRP, hypoalbuminemia, 
$\mathrm{FC}>50 \mu \mathrm{g} / \mathrm{g}$ and $>150 \mu \mathrm{g} / \mathrm{g}$ was significantly higher in IBD patients compared to non-IBD patients (40.0 vs $10.3 \%, p=0.002$, OR: $5.81,95 \%$, CI 1.77 to $19.06,25.0$ vs $5.9 \%, p=0.026$, OR: $5.33,95 \%$, CI 1.28 to 22.29 , 80.0 vs $39.7 \%, p=0.044$, OR: $6.07,95 \%$, CI 1.83 to 23.42 , and 60.0 vs $16.2 \%, p=0.002$, OR: $7.78,95 \%$, CI 1.83 to 20.14 respectively). The sensitivity, specificity, PPV, NPV, accuracy and AUROC of the AS, ALF, $\mathrm{FC}>50, \mathrm{FC}>150$ and their combinations are shown in Table II. AUROC for FC $>150$ and AS+ FC $>150$ were significant for predicting IBD (Figure 2) $(\mathrm{AUC}=0.715, p=0.011,95 \%$ CI: $0.566-0.865$ and $\mathrm{AUC}=0.702, p$ $=0.016,95 \%$ CI:0.541-0.863, respectively).

Fecal calprotectin (FC) was revealed to be a significant predictor in the discrimination of IBD and non-IBD diseases at a cutoff value of $207 \mu \mathrm{g} / \mathrm{g}(\mathrm{AUC}=0.794, p<0.05,95 \% \mathrm{CI}: 0.658-0.930)$, with a sensitivity and specificity of $70.6 \%$ and $82.5 \%$, respectively (Figure 3 ).

\section{Discussion}

The present study aimed to investigate the effectiveness of clinical and laboratory findings in the discrimination of IBD and other gastrointestinal diseases in pediatric patients. As proposed by Waugh et al., two distinct cutoff values were determined for FC $(50 \mu \mathrm{g} / \mathrm{g}$ and $150 \mu \mathrm{g} / \mathrm{g})(6)$. FC $<50 \mu \mathrm{g} / \mathrm{g}$ is known to rule out IBD (11). Holtman et al. reported that FC had a high sensitivity (0.99; 95\% CI, 0.81-1.00) and suggested that negative FC $(50 \mu \mathrm{g} / \mathrm{g})$ safely rules out IBD (10). Another study indicated that clinical follow-up is recommended in patients with an FC level of $50-150 \mu \mathrm{g} / \mathrm{g}$ and the patients with these levels may develop IBD as well as diseases that do not require endoscopy such as irritable bowel syndrome (IBS) (6). In such patients, additional findings are needed to make a decision for invasive procedures such as endoscopy.

The present study investigated the predictive role of AS and ALF in the diagnosis of IBD, among which involuntary weight loss was found to be the most significant predictor of IBD among AS. Heida et al. reported that rectal bleeding and perianal lesions were accepted as high-risk factors for the diagnosis of IBD while a family history of IBD, extraintestinal findings, and weight loss were accepted as low-risk factors (8). However, these low-risk factors were accepted as high-risk factors when combined with FC $>50 \mu \mathrm{g} / \mathrm{g}$. In our study, AS had a specificity of $39.71 \%$ and this level increased to $87.5 \%$ AS were combined with FC $>150$ $\mu \mathrm{g} / \mathrm{g}$.

Among the ALF analyzed, CRP and hypoalbuminemia were found to be more effective than other ALF in the diagnosis of IBD. Caviglia et al. reported that CRP was significantly higher in IBD patients compared to controls while FC was revealed as the only significant factor on multivariate regression analysis (12). Holtman et al. indicated that AS in combination with CRP had no remarkable benefit in the diagnosis of IBD while AS in combination with FC had the highest benefit (4). In our study, AS had a specificity of $72.06 \%$ when combined with ALF and a specificity of $87.5 \%$ when combined with FC $>50 \mu \mathrm{g} / \mathrm{g}$ Moreover, the accuracy rates and AUC indicated that ALF had no significant effect in the discrimination of IBD and non-IBD diseases.

In a previous meta-analysis, Degraeuwe et al. found the AUC value for FC $212 \mu \mathrm{g} / \mathrm{g}$ as 0.94 (95\% CI, 0.92-0.95) in the discrimination of IBD and non-IBD diseases (13). Another meta-analysis conducted in 2017 evaluated a large cohort of 1,120 pediatric patients and revealed that FC improved the AUC more than other laboratory markers (ESR, CRP, platelet count, hemoglobin, albumine). Additionally, the pooled AUC of FC (6 studies) was 0.95 (95\% 0.93-0.98) in this meta-analysis (9). In our study, the AUC for FC $207 \mu \mathrm{g} / \mathrm{g}$ was 0.794 , which was lower than those of other studies. As proposed by Holtman et al., there are numerous factors playing a role in the discrimination of IBD and non-IBD diseases. FC has a remarkably high AUC value, which could be associated with overestimation of FC levels. Moreover, the contribution of ALF to the AUC value is relatively lower due to the greater AUC values of AS and FC. On the other hand, the lower number of pediatric patients in meta-analyses further complicates this evaluation (9), which was a limitation of our study as well.

Given that FC testing is not available in most primary healthcare centers despite being a useful marker of IBD, the present study also aimed to provide a basis that could aid primary care clinicians in the referral of patients with suspicious IBD to pediatric gastroenterology clinics. Accordingly, AS and ALF are more 
important than FC, although they were found to be inadequate for the prediagnosis of IBD in the present study. In a previous study, $17(19 \%)$ out of 90 patients that had AS and were referred from a primary healthcare center were diagnosed with IBD (10). Similarly, in our patients, IBD was diagnosed in $25.4 \%$ of patients with AS. As a matter of fact, there are limited studies on this topic and thus further studies are needed to substantiate our findings.

\section{Conclusion}

Although primary care clinicians often use AS and laboratory parameters in the discrimination of IBD and non-IBD diseases, FC was found to have a relatively higher diagnostic value. Moreover, although there are varying cutoff values of $\mathrm{FC}$ reported in the literature, a cutoff value of $>150 \mu \mathrm{g} / \mathrm{g}$ was found to be highly effective in the diagnosis of IBD.

\section{References}

1. Lang T. Interfaces in Pediatric Gastrointestinal Endoscopy: Who Should Do It? Visc Med 2016; 32: 7-11.

2. Roseth AG, Schmidt PN, Fagerhol MK. Correlation between faecal excretion of indium-111-labelled granulocytes and calprotectin, a granulocyte marker protein, in patients with inflammatory bowel disease. Scand J Gastroenterol 1999;34:50-4.

3. Ton H, Brandsnes Dale S, Hotlund J, Skuibina F, Schjonsby H, et al. Improved assay for fecal calprotectin. Clin Chim Acta 2000;292:41-54.

4. Holtman GA, Lisman-van Leeuwen Y, Kollen BJ, Norbruis OF, Escher JC, Walhout LC, et al. Diagnostic test strategies in children at increased risk of inflammatory bowel disease in primary care. PLoS One. 2017 ; 12(12):e0189111.

5. Manceau H, Chicha-Cattoir V, Puy H, Peoc'h K. Fecal Calprotectin in Inflammatory Bowel Diseases: Update and Perspectives. Clin Chem Lab Med 2017;55(4):474-483.

6. Waugh N, Cummins E, Royle P, Kandala NB, Shyangdan D, Arasaradnam R, et al. f Health Technol Assess 2013;17:xv-xix, 1-211.

7. Van Rheenen PF, Van de Vijver E, Fidler V. Faecal calprotectin for screening of patients with suspected inflammatory bowel disease: diagnostic meta-analysis. Br Med J 2010;341:c3369.

8. Heida A, Vijver EV,Ravenzwaaij D,Biervliet SV,Hummel TZ, Yuksel Z, et al. Predicting inflammatory bowel disease in children with abdominal pain and diarrhoea: calgranulin-C versus calprotectin stool tests. Arch Dis Child 2018;103(6):565-571.

9. Holtman GA, Lisman-van Leeuwen Y, Day AS, Fagerberg UL, Hendersen P, Leach ST et al. Use of Laboratory Markers in Addition to Symptoms for Diagnosis of Inflammatory Bowel Disease in Children: A Meta-analysis of Individual Patient Data. JAMA Pediatr . 2017;171(10):984-991.

10. Holtman GA, Lisman-van Leeuwen Y, Kollen BJ, Norbruis OF, Escher JC, Kindermann A, et al. Diagnostic Accuracy of Fecal Calprotectin for Pediatric Inflammatory Bowel Disease in Primary Care: A Prospective Cohort Study. Ann Fam Med 2016; 14(5):437-445.

11. Gisbert JP, McNicholl AG. Questions and answers on the role of faecal calprotectin as a biological marker in inflammatory bowel disease. Dig Liver Dis 2009; 41: 56-66.

12. Caviglia GP, Pantaleoni S, Touscoz GA, et al. Fecal calprotectin is an effective diagnostic tool that differentiates inflammatory from functional intestinal disorders. Scand J Gastroenterol . 2014;49(12):14191424 .

13. Degraeuwe PL, Beld MP, Ashorn M, et al. Faecal calprotectin in suspected paediatric inflammatory bowel disease. J Pediatr Gastroenterol Nutr . 2015;60(3):339-346.

\section{Figure Legends}

Figure 1. Final diagnosis of patients after colonoscopic examination

Figure 2. Fecal calprotectin, alarm symptoms and abnormal laboratory findings for predicting IBD

Figure 3. Fecal calprotectin for predicting IBD (AUC=0.794, $<<0.05,95 \%$ CI: $0.658-0.930$ ) at a cutoff 
value of $207 \mu \mathrm{g} / \mathrm{g}$.

\section{Hosted file}

Table I.docx available at https://authorea.com/users/359882/articles/481641-impact-of-fecalcalprotectin-measurement-for-the-diagnosis-of-inflammatory-bowel-disease-in-childrenwith-alarm-symptoms

\section{Hosted file}

Table II.docx available at https://authorea.com/users/359882/articles/481641-impact-offecal-calprotectin-measurement-for-the-diagnosis-of-inflammatory-bowel-disease-inchildren-with-alarm-symptoms

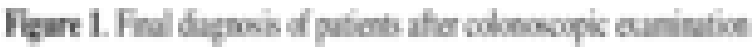

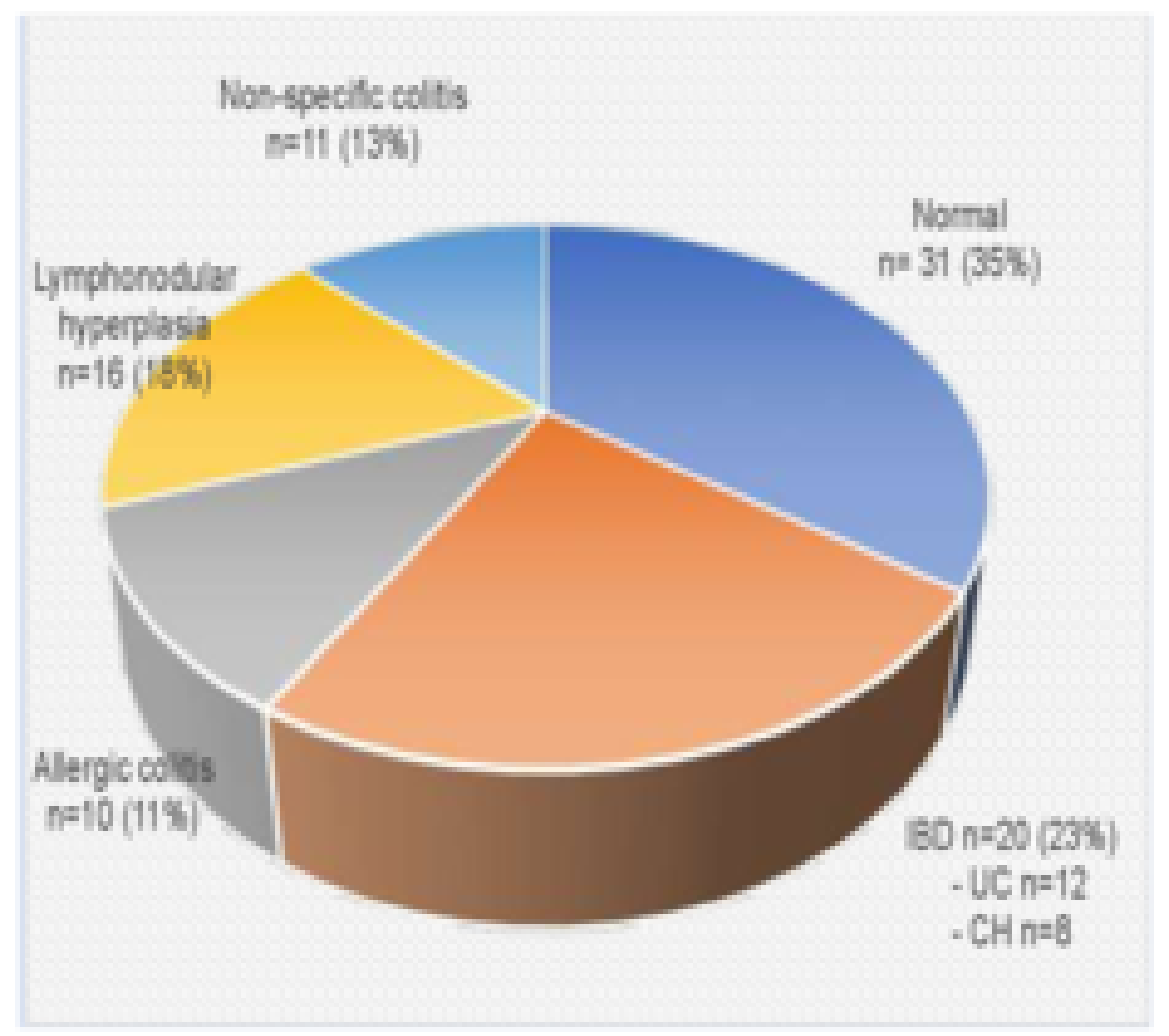




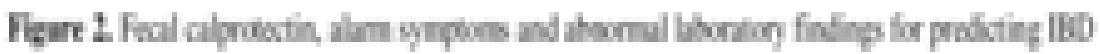

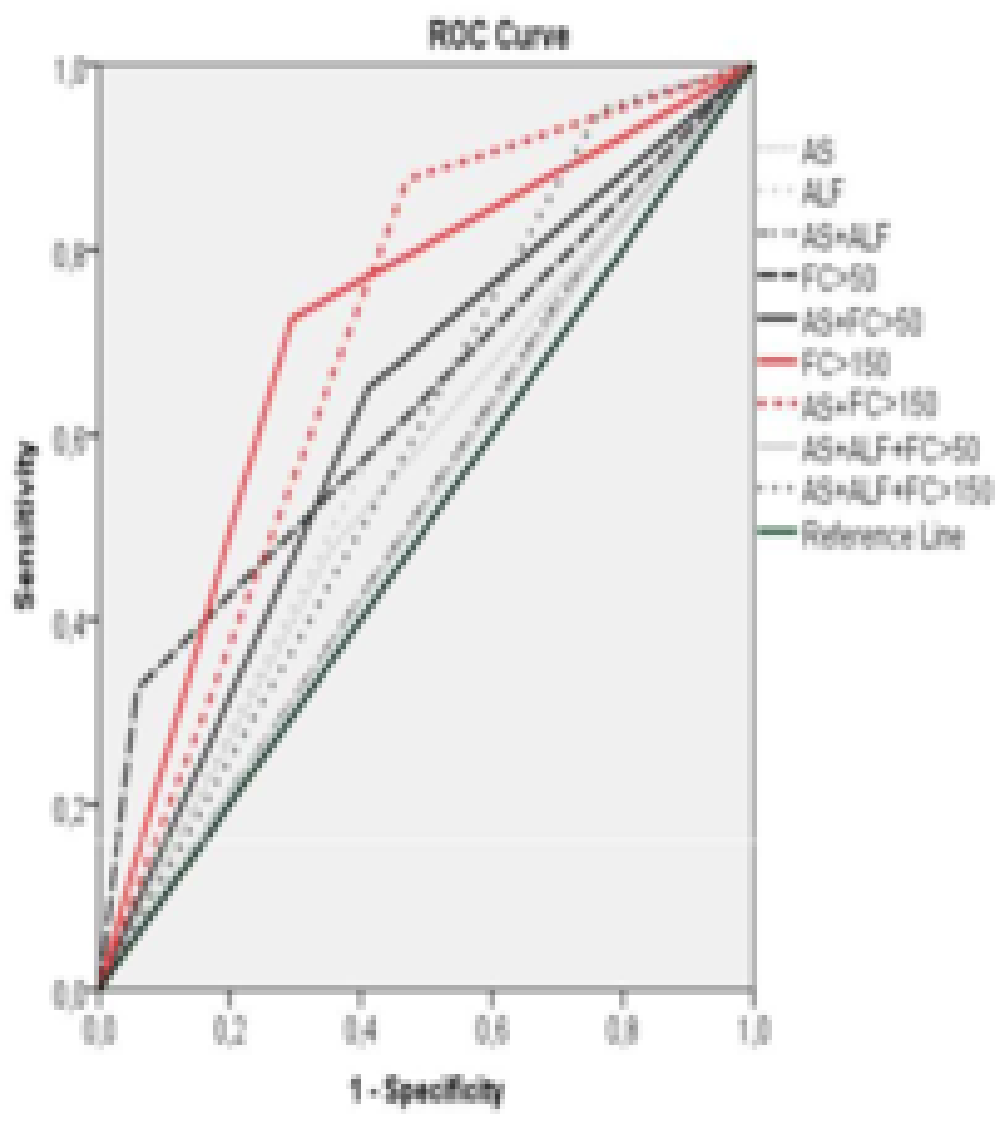

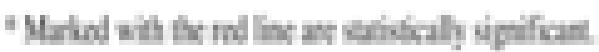

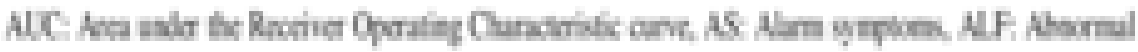

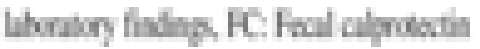




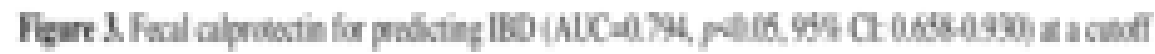

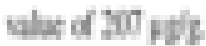

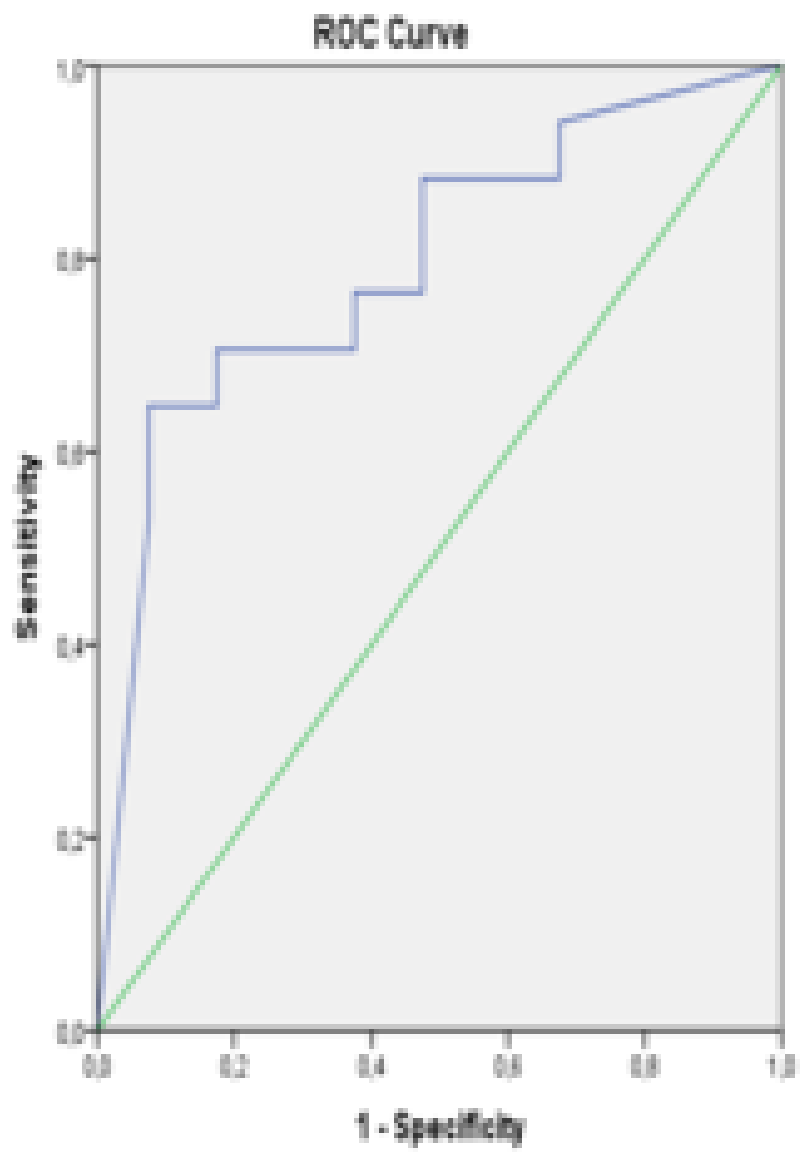

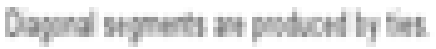

